\title{
Assessment of late pilot injection effect in dual- fuel combustion
}

\author{
Antonio Caricato ${ }^{1, *}$, Antonio Paolo Carlucci ${ }^{1}$, Antonio Ficarella ${ }^{1}$, and Luciano Strafella ${ }^{1}$ \\ ${ }^{1}$ Department of Engineering for Innovation, University of Salento, Lecce, 73100, Italy
}

\begin{abstract}
In this paper, the effect of late injection on combustion and emission levels has been investigated on a single cylinder compression ignition engine operated in dual-fuel mode injecting methane along the intake duct and igniting it through a pilot fuel injected directly into the combustion chamber. During the tests, the amount of pilot fuel injected per cycle has been kept constant, while the amount of methane has been varied on three levels. Therefore, three levels of engine load have been tested, while speed has been kept constant equal to $1500 \mathrm{rpm}$. Pilot injection pressure has been varied on three set points, namely 500, 1000 and 1500 bar. For each engine load and injection pressure, pilot injection timing has been swept on a very broad range of values, spanning from very advanced to very late values. The analysis of heat release rate indicates that MK-like conditions are established in dual-fuel mode with late pilot injection. In these conditions, pollutant species, and NOx levels in particular, are significantly reduced without penalization - and in several conditions with improvement - on fuel conversion efficiency.
\end{abstract}

\section{Introduction}

The natural gas is a mixture of methane, ethane, propane, butanes, pentanes and other hydrocarbons. It formed from decayed animals and plants million years ago, built up in thick layers and trapped beneath the surface of the earth. Over time, intense heat and pressure changed these fossils into black oils, coals and natural gases. Natural gas is extracted from the underground formation through a well with other liquid hydrocarbons and nonhydrocarbons, which later are filtered from these components and sent through pipelines for distribution. Technology advances have enabled in the domestic energy the use of methane, which rapidly grow in Canada, China, Europe and the USA.

A diesel engine fumigated with natural gas, or methane is called a diesel derivative dualfuel engine. Even though internal combustion engines (ICE) running on natural gas are characterized by lower $\mathrm{NO}_{\mathrm{x}}$ and particulate matter (PM) emissions levels, they are generally penalized in terms of $\mathrm{CO}$ and $\mathrm{HC}$ especially at part load compared to conventional diesel engine $[1,2]$. The combustion is slower at part load while faster at higher load.

The substitution rate, defined as the ratio of natural gas energy to the sum of natural gas and diesel energy can overcome $90 \%$ in the currently available dual-fuel engines, so significantly reducing $\mathrm{CO}_{2}$ and particulate matter (PM) emission levels. However, besides the advantages of dual fuel combustion, some well-known drawbacks also arise, as the knock tendency at high loads, and at low loads a high production of carbon monoxide (CO).

Homogeneous charge compression ignition (HCCI) combustion can be a viable solution to reduce drastically these problems [2], [3]. In HCCI operation air and fuel are

\footnotetext{
*Corresponding author: antonio.caricato1@unisalento.it
} 
homogeneously mixed before being ignited through compression. In this way, fuel rich zones are avoided, local temperatures are reduced, and, overall, emissions are drastically decreased. In dual-fuel diesel-methane engines, as previously said, the ignition of a mixture of air and methane is obtained thanks to an injection of diesel fuel. Implementing HCCI in dual-fuel diesel-methane engines, therefore, requires to obtain a homogeneous mixture of air and methane as well as homogeneous distribution of diesel fuel vapor inside the cylinder before the start of ignition. While mixing homogeneously air and methane is a relatively easy task, the same cannot be said about diesel fuel. Several attempts have been documented in which the diesel fuel is fumigated along the intake duct. However, this solution, while guaranteeing a proper homogenization of the diesel fuel with air before ignition, presents as drawbacks the high PM emission levels due to poor fuel atomization conditions [4].

Many strategies, proposed in literature, were evaluated with the goal of increasing the mixture ignition delay in dual-fuel conditions and so giving more time to the diesel fuel to homogeneously spread, vaporize and mix with air. Pilot start of injection (SOI), pilot injection pressure (p_rail), pilot injection splitting, substitution rate, engine compression ratio (CR), intake charge pressure (ICP) and temperature (ICT), exhaust gas recirculation (EGR), and pilot fuel chemical composition resulted the parameters most influential on ignition delay.

Ryu [5,6] studied the NG dual-fuel combustion varying SOI in the range 11-23 Crank Angle Degrees before Top Dead Center (CAD BTDC) and p_rail from 30 to $150 \mathrm{MPa}$, concluding that: 1) the increase of pilot injection pressure results in lower smoke, but higher NOx emission; 2) smoke is decreased and NOx is increased as the pilot injection timing is advanced. Compared to diesel-only combustion, however, smoke emissions are significantly reduced over the range of operating conditions and NOx emissions are decreased over the range with the exception of the full load case.

Guerry et al. in (2016) [7] varied SOI on a wide range of values, testing a single-cylinder compression ignition engine, observing that, when SOI was advanced, the ignition delay (ID) and the separation between the end of injection and start of combustion both increased. The heat release rate (HRR) curve changed from a distinct two-stage profile to a smooth singlestage profile.

Ibrahim et al. in (2015) [8], testing a single-cylinder compression ignition engine operated in dual-fuel diesel-biogas mode, demonstrated that, acting on SOI, ICT, and substitution rate, the engine could be effectively operated in PPCCI at low/medium loads with a beneficial reduction of nitric oxides (NOx) and THC with respect to conventional DF combustion mode.

Carlucci at al. in (2017) [9] describe the results of a full factorial DoE in which the effects of compression ratio, intake charge pressure (ICP), pilot injection timing and pressure, and methane flow rate effect are evaluated and discussed on combustion development, engine performance, and pollutant emission levels at the exhaust. Through analysis of variance (ANOVA), the first- and second-order effects were also quantified. Moreover, the factor variation ranges leading the engine to operate in HCCI or PPCCI (Partially Premixed Charge Compression Ignition) combustion, i.e., guaranteeing a high conversion efficiency and low emission levels at the same time, were sought and highlighted. This suggested that not only very advanced but also retarded injection timings, combined with high ICP, determine very low levels of nitrogen oxides, with little or no penalty on engine.

Based on these last results, aim of this work is to better assess, on the same engine and experimental setup of [9], the effect of late injection on engine performance and emission levels. This combustion concept has been already investigated in literature with only diesel fuel [10]. It has been named Modulated Kinetic (MK) and allowed to establish homogeneous air/diesel fuel mixing through a proper calibration of injection timing and pressure, swirl, hot/cold EGR and compression ratio. The same approach is proposed in this paper, operating the engine in dual-fuel conditions. For each operating conditions, the engine behavior has 
been fully characterized. In particular, in-cylinder pressure, air, diesel fuel and methane flow rates, pollutant emission levels at the exhaust have been measured. Based on the above measurements, gross heat release rate, indicated mean effective pressure, specific fuel consumption and emission levels have been calculated.

The analysis of heat release rate indicates that MK-like conditions are established in dualfuel mode with late pilot injection. In these conditions, pollutant species, and NOx levels in particular, are significantly reduced without penalization - and in several conditions with improvement - on fuel conversion efficiency.

\subsection{Basic Concept of MK concept to dual-fuel combustion}

The basic concept of MK combustion on dual fuel engine is schematically explained in Fig.1. Because $\mathrm{NO}_{\mathrm{x}}$ is strongly dependent on the combustion temperature, one effective way to reduce $\mathrm{NO}_{\mathrm{x}}$ emission is to lower the combustion temperature, for example by applying heavy EGR or decrease compression ratio. Unfortunately, this leads to a decrease of engine performance.

Since reducing oxygen concentration results in misfiring or high $\mathrm{HC}$ level, a challenge to obtain a reduction of pollution was made a premixed combustion process using various methodologies, where the fuel and oxygen must be thoroughly mixed prior to ignition. A sufficient mixing time is secured by prolonging the ignition delay that was obtained by using a significantly retarded fuel injection timing and increasing swirl ratio. However, the retarded injection timing may yield a lowered cycle efficiency and increased $\mathrm{HC}$ emission; generally smoke level in dual fuel engine, if compered to diesel engine are very low, because the amount of diesel fuel quantity is lower than in the standard diesel engine [10].

The distribution of fuel /air and methane mixture in cylinder could be optimized by choosing a high swirl ratio to suppress $\mathrm{HC}$ and to reduce the cooling losses from burning gases to the combustion chamber walls.

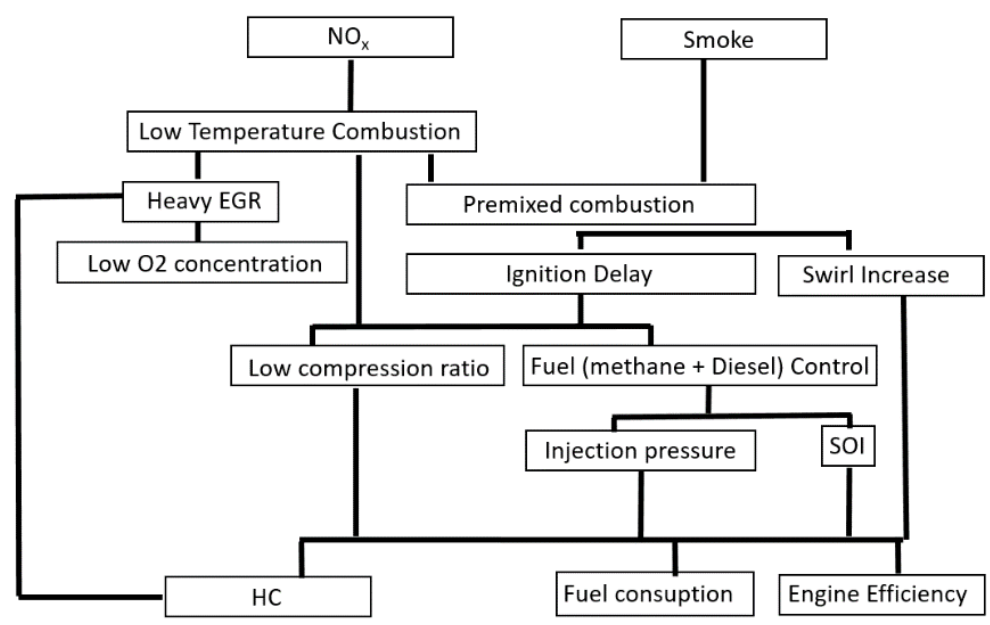

Fig. 1. MK concept implementation in dual-fuel diesel-methane conditions 


\section{Experimental method}

To perform the tests, the same experimental layout illustrated in [9] was used. A compression ignition single cylinder, 4-stroke, common-rail Diesel research engine (AVL model 5402) was used to analyze the effect of pilot injection pressure and timing. The main technical features of the engine are reported in Tab 1, while in Fig 2 the scheme of the experimental layout is reported. The gaseous methane has been fumigated along the intake duct of the engine by means of an injector consisting of an automatic poppet valve, with contrast spring, actuated by the methane itself introduced by a solenoid valve in a small accumulation volume (more details about the injection system can be found in [9]). The solenoid valve was actuated using a duty cycle $\mathrm{D}=50 \%$ with a frequency of $30 \mathrm{~Hz}$. The amount of methane introduced along the intake duct was varied acting on the duty cycle of a continuous Pulse-Width Modulation (PWM) signal, generated by a National Instruments High-Speed Sourcing Digital Output NI 9474 module and controlled by means of LabView software. In this way the injection time of the gas injector was varied and so the amount of adducted methane. During the tests, the injector was positioned at a distance of about $400 \mathrm{~mm}$ from the cylinder axis, so that a homogeneous methane-air mixture was obtained before trapping it into the cylinder. Although the engine is provided with a swirl and tumble valves, the mixture was introduced in the cylinder through the swirl valve only. This solution was chosen because it provided the best compromise in terms of engine performance and emission level [11]. Tab 2 reports the levels assigned to each factor varied during tests. The duration of the diesel fuel injection was adjusted in order to keep constant - equal to $6 \mathrm{~mm} 3 /$ cycle - the diesel fuel amount injected per cycle. In particular, a duration equal to 150 or $300 \mu \mathrm{s}$ was set for a diesel fuel injection pressure (p_rail) respectively equal to 1500,1000 or 500 bar.

The engine was always run at $1500 \mathrm{rpm}$. Either referring to SOI variation range, more advanced SOI values were not used because of the high MPRR observed in several conditions, not tolerable by the engine structure. On the other hand, results obtained with more retarded SOI are not shown, as the combustion was not observed.

Table 1. Main specification of single cylinder diesel engine.

\begin{tabular}{lll}
\hline Specification & Nominal value/description \\
\hline Maximum Power & $18 \mathrm{~kW}$ \\
Bore & $85 \mathrm{~mm}$ \\
Stroke & $90 \mathrm{~mm}$ \\
Original Compression ratio & $17.1: 1$ \\
Combustion chamber & Bowl with valve pockets and flat head \\
Injection system & Common Rail & \\
Max Injection pressure & $1600 \mathrm{bar}$ & \\
Valve timing & Opening & Closing \\
Intake & $13.5^{\circ} \mathrm{BTDC}$ & $46.5^{\circ} \mathrm{ABDC}$ \\
Exhaust & $51.5^{\circ} \mathrm{BBDC}$ & $16.5^{\circ} \mathrm{ATDC}$ \\
\hline
\end{tabular}




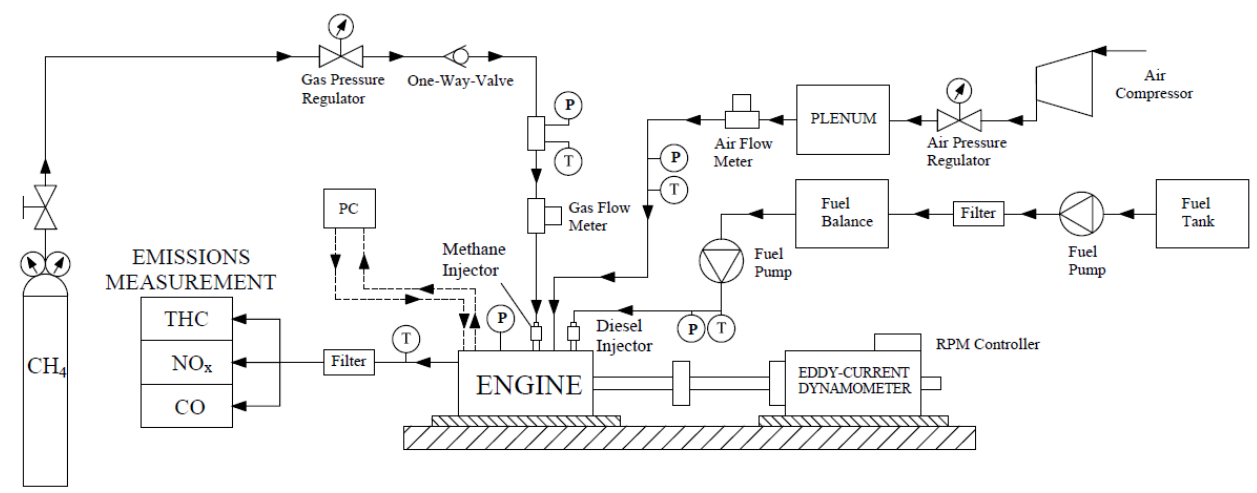

Fig. 2. Experimental layout.

Table 2 - Factors and corresponding levels varied during tests.

\begin{tabular}{lll}
\hline Description & Abbreviation & Tested levels \\
\hline & & 6 SLM - low level (a) \\
Methane mass flow rate & Ggas & 23 SLM - medium level (b) \\
& & 30 SLM- high level (c) \\
Diesel fuel injection pressure & p_rail & $500-1000-1500[\mathrm{bar}]$ \\
Diesel fuel pilot injection timing & SOI & $-50 /-35 /-20 /-10 / 0 / 5 / 10$ \\
& & {$[$ CAD ATDC] } \\
\hline
\end{tabular}

Data acquisition was started only after steady conditions were reached. An AVL piezoelectric pressure sensor model QC33C was mounted in the combustion chamber. The signal sampling was triggered and synchronized with TDC by an AVL encoder model 364C with a resolution of 0.2 crank angle degrees (CAD). The in-cylinder pressure transducer was characterized by a linearity error lower than $0.4 \%$, while the sensitivity shift and the range error of the pressure amplifier (AVL model 3066A01) were less than 1\%. The error associated to the $\mathrm{A} / \mathrm{D}$ data conversion and acquisition was equal to $0.3 \%$. To obtain the absolute cylinder pressure $\left(p_{c y l, a b s}\right)$, a pegging routine was used through which the cylinder pressure measured by the in-cylinder pressure during the intake stroke was imposed equal to the average pressure measured by an absolute pressure sensor (Kistler piezoresistive sensor type 4045A2) mounted along the intake duct. Then, the absolute cylinder pressure so obtained was stored for 50 consecutive cycles, averaged and then post-processed. The gross HRR was calculated using the same method of [9].

The estimated error in this calculation, due to the errors in the absolute cylinder pressure measurement, is around $2 \%$. Based on $p_{c y l, a b s}$, it was possible to estimate the indicated power $P_{\mathrm{i}}$ supplied by the engine:

$$
P_{i}=\frac{n}{n_{R}} \oint_{\text {cycle }} p_{c y l, a b s} d V
$$


where $n$ is the engine speed while $n_{R}(=2$ in this case) is the number of crank revolutions for each power stroke. The average diesel fuel consumption was measured by means of an AVL 733S Fuel Balance. The methane flux was stabilized by means of a $0.8 \mathrm{~L}$ plenum positioned upstream the methane injector. The average methane fuel consumption $\mathrm{G}_{\mathrm{gas}}$ was measured by means of a thermal mass flow Aalborg Instruments and Controls Inc., model DFC 36 characterized by a measurement error below $1 \%$.

The measurement of the fuels consumption allowed to estimate the fuel conversion efficiency referred to the indicated power, $\eta_{f}$ :

$$
\eta_{f}=\frac{P_{i}}{\dot{m}_{d} H_{i, d}+G_{g a s} H_{i, \text { gas }}}
$$

Where $H_{i, d}$ and $H_{i, g a s}$ are the lower calorific value of diesel fuel and methane respectively. The combustion efficiency was calculated as:

$$
\eta_{b}=1-\frac{\sum x_{i} H_{i, i}}{\left(\frac{\dot{m}_{d} H_{i, d}+G_{g a s} H_{i, g a s}}{\dot{m}_{a}+\dot{m}_{d}+G_{g a s}}\right)}
$$

In which $\mathrm{x}_{\mathrm{i}}$ is the mass fractions of $\mathrm{CO}$ and THC measured at the exhaust, $\mathrm{H}_{\mathrm{i}, \mathrm{i}}$ the related lower calorific values, and $\mathrm{m}_{\mathrm{a}}$ is the consumed intake air mass measured by means of an AVL Flowsonix mounted between two plenums in order to stabilized the air flux. Because the composition of THC in the exhaust, and thus the related $\mathrm{H}_{\mathrm{i}, \mathrm{i}}$, is not known, the combined mass-fraction-weighted $\mathrm{H}_{\mathrm{i}}$ of diesel fuel and methane has been used to represent the lower calorific value of THC.

The efficiency of the thermodynamic cycle was calculated as:

$$
\eta_{c}=\frac{\eta_{f}}{\eta_{b}}
$$

The combustion products and therefore the pollutant emissions levels at the engine exhaust have been measured by means of an AVL AMA i60 Exhaust Measurement System.

The combustion products and therefore the pollutant emissions levels at the engine exhaust have been measured by means of an AVL AMA i60 Exhaust Measurement System. In particular, THC levels have been measured through an AVL Flame Ionization Detector (FID) analyzer, whose operation is based on the measurement of the electrical current yield by the organic carbon atoms ionized in a hydrogen flame. $\mathrm{NO}_{\mathrm{x}}$ levels were measured through on AVL Chemiluminescence Detector (CLD) analyzer, whose operation is based on the measurement of the light emitted during the oxidation of nitrogen oxide $\mathrm{NO}$ with ozone $\mathrm{O}_{3}$, and CO levels have been measured through an Infrared Detector (IRD) analyzer, whose operation is based on the measurement of an infrared radiation absorption, correlated to the concentration of the component to be measured.

Particulate matter at the tailpipe was not measured since, as known, the utilization of methane drastically reduces this pollutant, well below $1 \mathrm{mg} / \mathrm{m}^{3}$. [9] 


\section{Results and discussion}

Pressure injection is analyzed to understand the effect of droplet fuel dimensions, spray penetration and fuel vaporization inside combustion chamber with air and fuel mixture.

Tab 3 indicates the injection settings for which misfiring was observed varying methane amount. It can be noticed that misfire was detected at injection pressure of 1000 and 1500 bar with very advanced SOI (-45 CAD BTDC) and late SOI (2.5 CAD ATDC). This result can be due to the fact that, given the low amount of diesel pilot fuel injected at high injection pressure, a faster vaporization and a better fuel-air mixing are expected. This leads to a reduction of the stoichiometric air-to-fuel ratio zones where ignition could takes place. This effect, already highlighted in literature [9] is emphasized for medium and low amount of methane.

The key points discussed in this study are the effect of SOI on combustion development at different methane amounts and engine efficiency, as described in the following sections.

Table 3. Design of experiment and misfire detection

(a)

\begin{tabular}{ccc}
\hline $\begin{array}{c}\text { SOI } \\
\left.{ }^{\circ}\right)\end{array}$ & $\begin{array}{c}\text { Injection } \\
\text { pressure } \\
\text { (bar) }\end{array}$ & Fire \\
\hline-45 & 500 & $\mathrm{~N}$ \\
-25 & 500 & $\mathrm{Y}$ \\
$-2,5$ & 500 & $\mathrm{Y}$ \\
0 & 500 & $\mathrm{Y}$ \\
2,5 & 500 & $\mathrm{~N}$ \\
-45 & 1000 & $\mathrm{~N}$ \\
-25 & 1000 & $\mathrm{~N}$ \\
$-2,5$ & 1000 & $\mathrm{Y}$ \\
0 & 1000 & $\mathrm{Y}$ \\
2,5 & 1000 & $\mathrm{~N}$ \\
-45 & 1500 & $\mathrm{~N}$ \\
-25 & 1500 & $\mathrm{~N}$ \\
$-2,5$ & 1500 & $\mathrm{Y}$ \\
0 & 1500 & $\mathrm{Y}$ \\
2,5 & 1500 & $\mathrm{~N}$ \\
\hline
\end{tabular}

(b)

\begin{tabular}{ccc}
\hline $\begin{array}{c}\text { SOI } \\
\left({ }^{\circ}\right)\end{array}$ & $\begin{array}{c}\text { Injection } \\
\text { pressure } \\
\text { (bar) }\end{array}$ & Fire \\
\hline-45 & 500 & $\mathrm{Y}$ \\
-25 & 500 & $\mathrm{Y}$ \\
$-2,5$ & 500 & $\mathrm{Y}$ \\
0 & 500 & $\mathrm{Y}$ \\
2,5 & 500 & $\mathrm{Y}$ \\
-45 & 1000 & $\mathrm{~N}$ \\
-25 & 1000 & $\mathrm{Y}$ \\
$-2,5$ & 1000 & $\mathrm{Y}$ \\
0 & 1000 & $\mathrm{Y}$ \\
2,5 & 1000 & $\mathrm{~N}$ \\
-45 & 1500 & $\mathrm{~N}$ \\
-25 & 1500 & $\mathrm{Y}$ \\
$-2,5$ & 1500 & $\mathrm{Y}$ \\
0 & 1500 & $\mathrm{~N}$ \\
2,5 & 1500 & $\mathrm{~N}$ \\
\hline
\end{tabular}

(c)

SOI Injection Fire

$\left(^{\circ}\right)$ pressure

\begin{tabular}{ccc} 
& (bar) & \\
\hline-45 & 500 & $\mathrm{Y}$ \\
-25 & 500 & $\mathrm{Y}$ \\
$-2,5$ & 500 & $\mathrm{Y}$ \\
0 & 500 & $\mathrm{Y}$ \\
2,5 & 500 & $\mathrm{Y}$ \\
-45 & 1000 & $\mathrm{~N}$ \\
-25 & 1000 & $\mathrm{Y}$ \\
$-2,5$ & 1000 & $\mathrm{Y}$ \\
0 & 1000 & $\mathrm{Y}$ \\
2,5 & 1000 & $\mathrm{Y}$ \\
-45 & 1500 & $\mathrm{~N}$ \\
-25 & 1500 & $\mathrm{Y}$ \\
$-2,5$ & 1500 & $\mathrm{Y}$ \\
0 & 1500 & $\mathrm{Y}$ \\
2,5 & 1500 & $\mathrm{Y}$ \\
\hline
\end{tabular}

\subsection{Effect of SOI and injection pressure on combustion development at different methane amounts}

In this section, the effect of SOI variation is analyzed. Figure 3 shows the HRR peak calculated on the basis of cylinder pressure at $1500 \mathrm{rpm}$ for all different pilot timings (varied from -45 CAD BTDC up to +2.5 CAD ATDC) and all injection pressure values. Tests have 
been run injecting always the same amount of diesel fuel $\left(6 \mathrm{~mm}^{3} /\right.$ cycle $)$ at various methane amounts: in detail, with low (a), medium (b) and high methane amount (c).

Based on engine behavior, two different SOI ranges are identified:

- The first range is for SOI from -45 CAD ATDC to -25 CAD BTDC (advanced SOI). The heat released during combustion has the same behaviour of a premixed combustion with the presence, close to the end of combustion, of a small diffusive combustion, as shown in Fig.3. This is due to the characteristic of dual-fuel combustion: thanks to good premixing due the low temperature at which the injection is performed, when engine reaches the temperature and pressure conditions for auto ignition, the heat release rate is very high. This behaviour leads to a high pressure inside the cylinder;

- The second range, defined from -2.5 CAD ATDC to 2.5 CAD BTDC (late SOI), is characterized by a HRR shape closer to the one observed in conventional diesel combustion: a premixed peak followed by a queue with a lower HRR (Figure 3). In this case, this second combustion phase is more pronounced as the methane introduced in cylinder was increased (Figure 3, plots b and c). Indeed, the idea of this study is to better analyse this SOI range where MK condition could be reached.

Referring to Fig. 3 (c), HRR peak first increases as SOI is retarded from very advanced SOI, then decreases as SOI is further retarded after TDC. This trend is still observed as the methane rate decreases (Fig. 3, plots a and b).

(a)

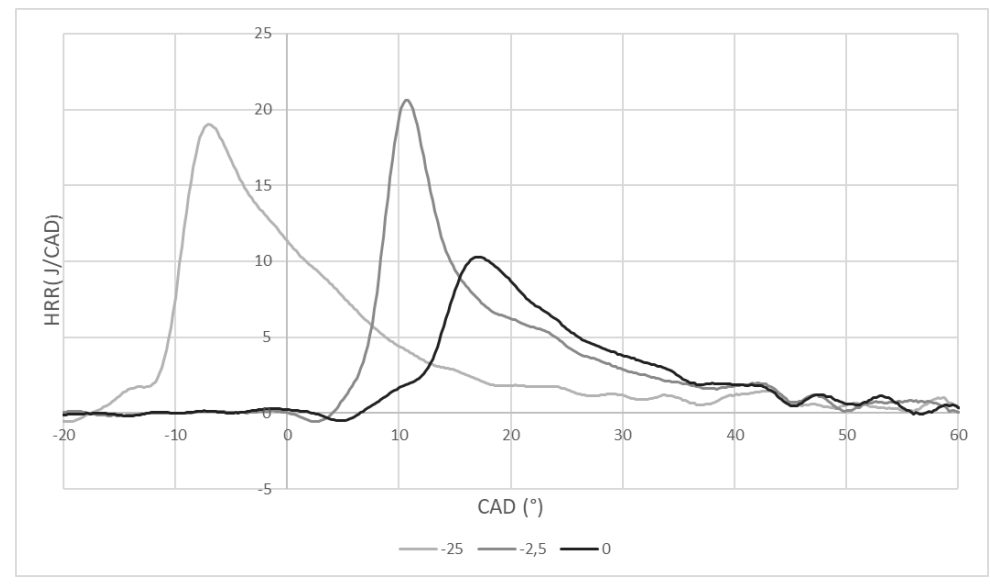

(b)

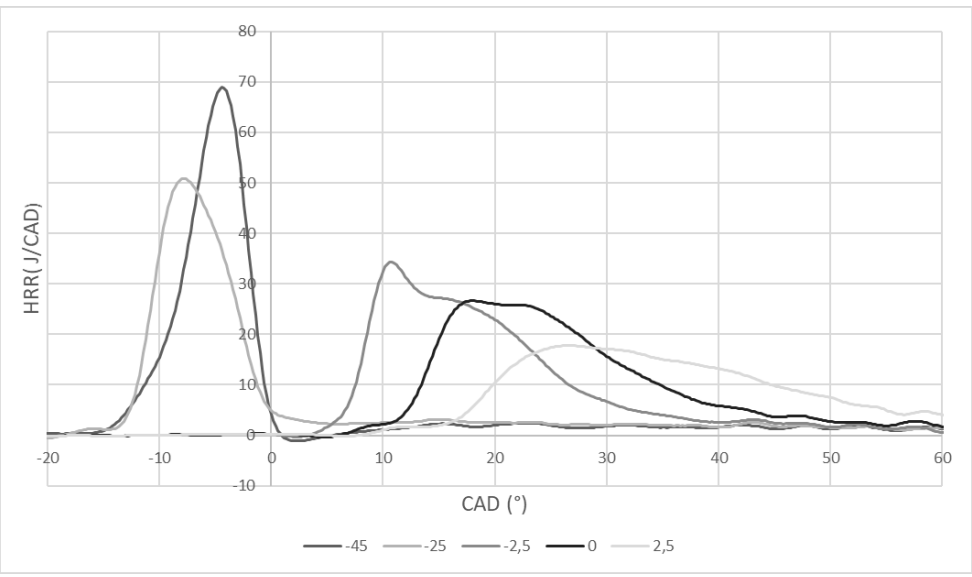


(c)

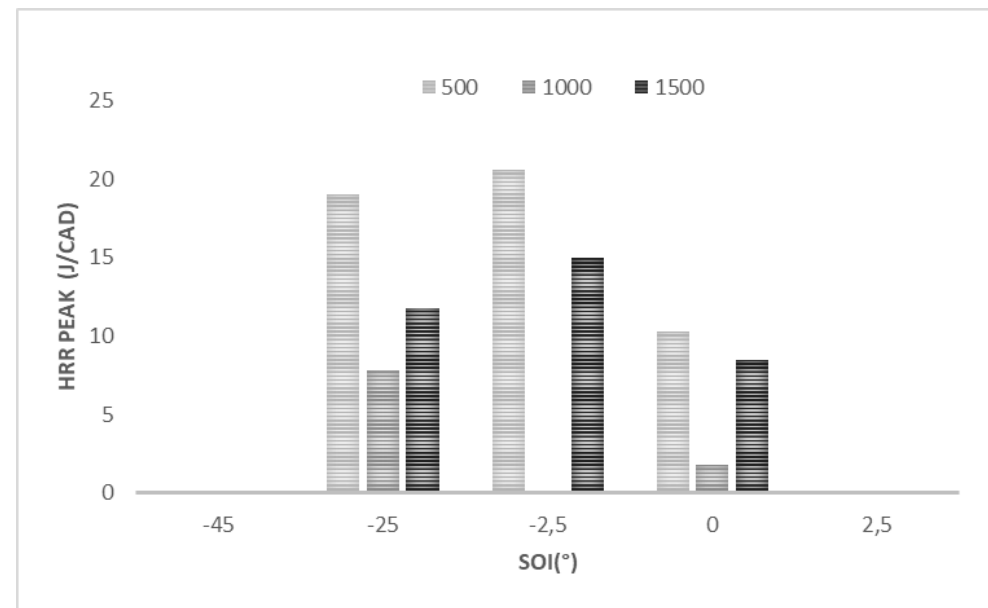

Fig. 3. HRR curves at 500 and $\mathrm{a}, \mathrm{b}$ and $\mathrm{c}$ amount of methane.

Figure 4 shows how methane rate influences HRR peak and CAD corresponding to maximum HRR. As methane rate increases, the HRR peak increases. For any methane rate, as SOI advances, ignition delay is prolonged and this can lead to overleaning of the mixture and low combustion rate, as shown in Figure 5. On the other hand, as SOI is retarded, ignition condition may not be reached during to the expansion phase (+2.5 CAD ATDC).

At SOI equal to 2.5 CAD BTDC, a strong reduction of HRR peak is observed due to engine combustion instability that generates alternatively burning or misfiring. Furthermore, the value of peak is lower as pressure injection increase at low methane amount and increase as pressure increase at high methane amount.

Results shown in Figure 4 and Figure 5 are coherent with Carlucci et al. study [9].

(a)

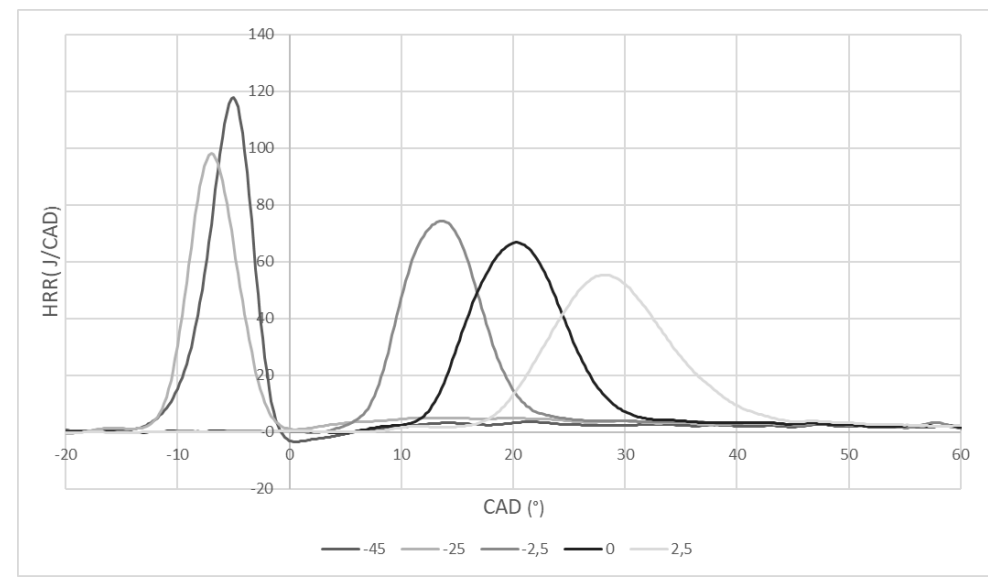


(b)

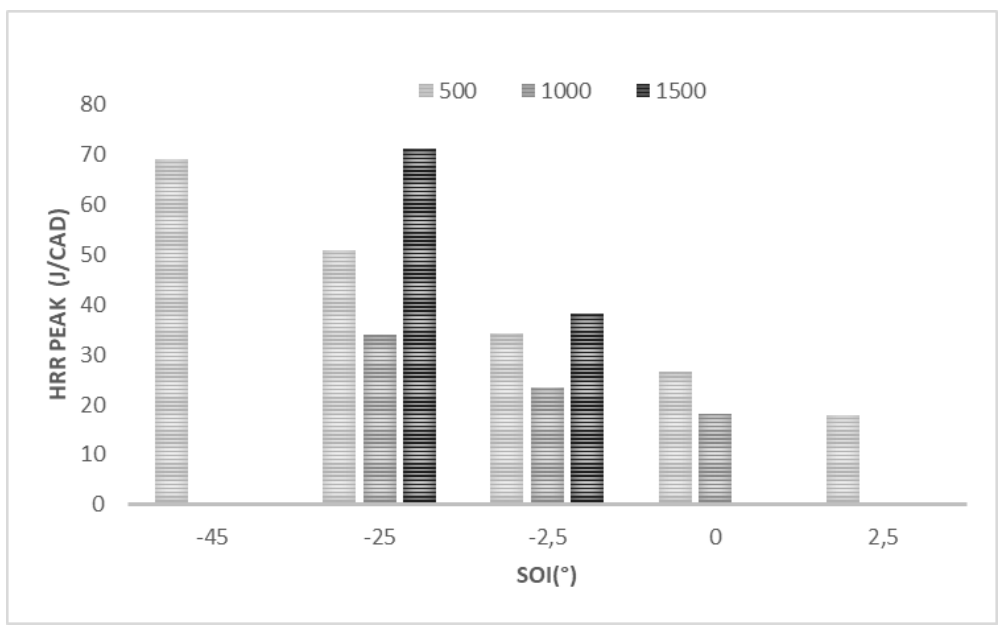

(c)

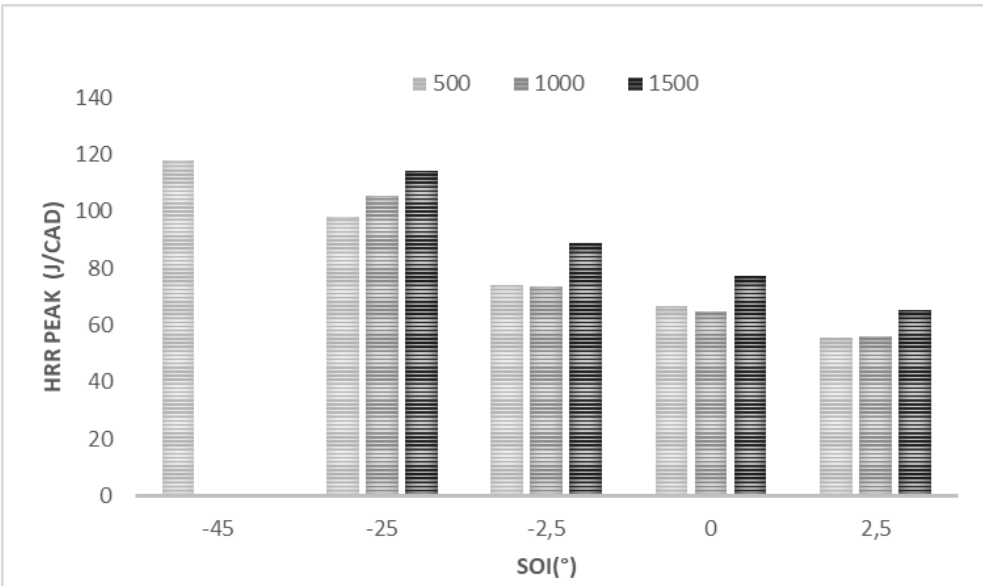

Fig. 4. HRR peak and SOI-HRR peak at 500, 1000 and 1500 bar and a, b and c amount of methane

(a)

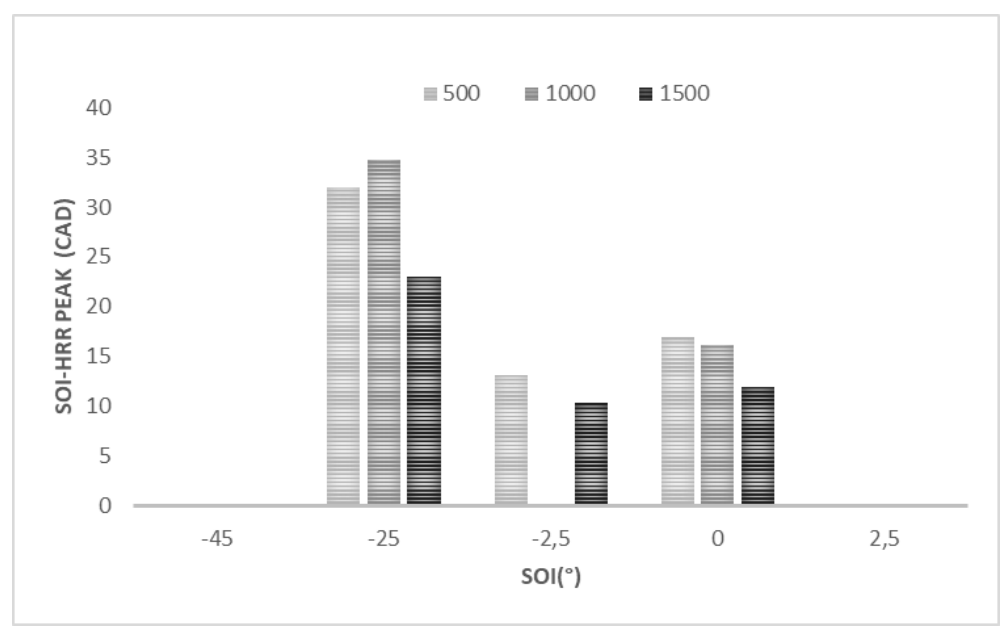


(b)

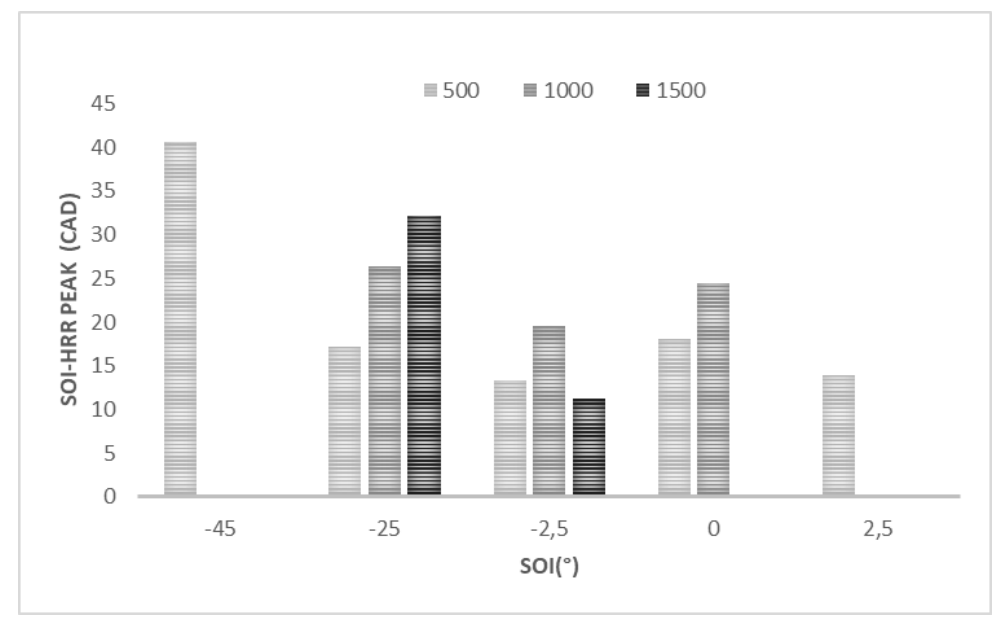

(c)

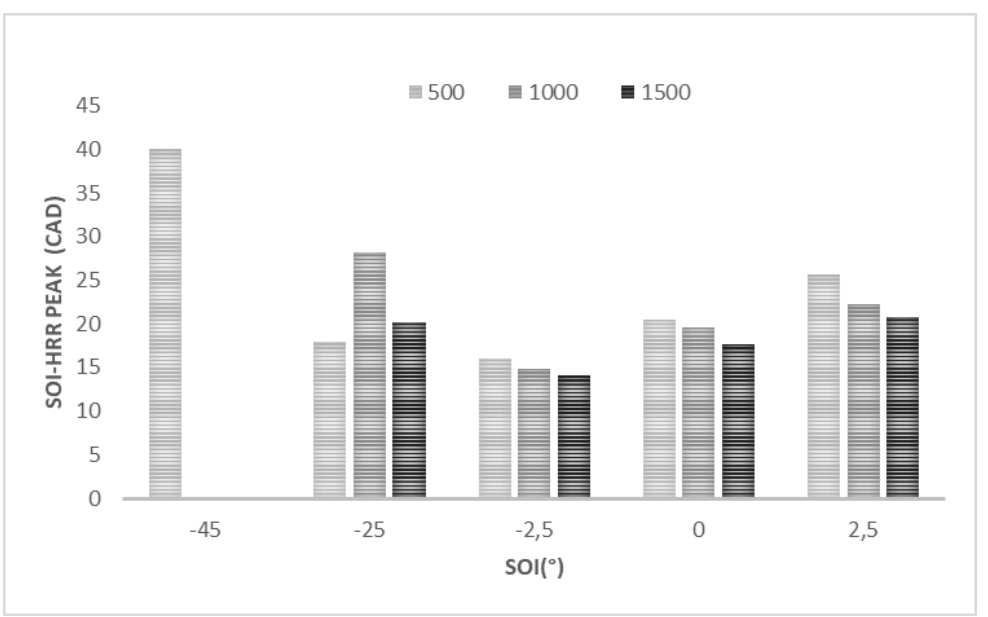

Fig. 5. SOI-HRR peak at 500, 1000 and 1500 bar and a, b and c amount of methane

Figure 6 highlights the effects of SOI and pressure injection on emissions at high methane amount (condition $c$ ).

Referring to 500 bar and c) condition, $\mathrm{NO}_{\mathrm{x}}$ emissions decrease when $\mathrm{SOI}$ increases.

On the other side, $\mathrm{HC}$ production is almost constant but $\mathrm{CO}_{2}$ production follows $\mathrm{NO}_{\mathrm{x}}$ trend. Furthermore, as pressure injection increases, all pollution species shown in Fig. 6 increase at the same SOI. 

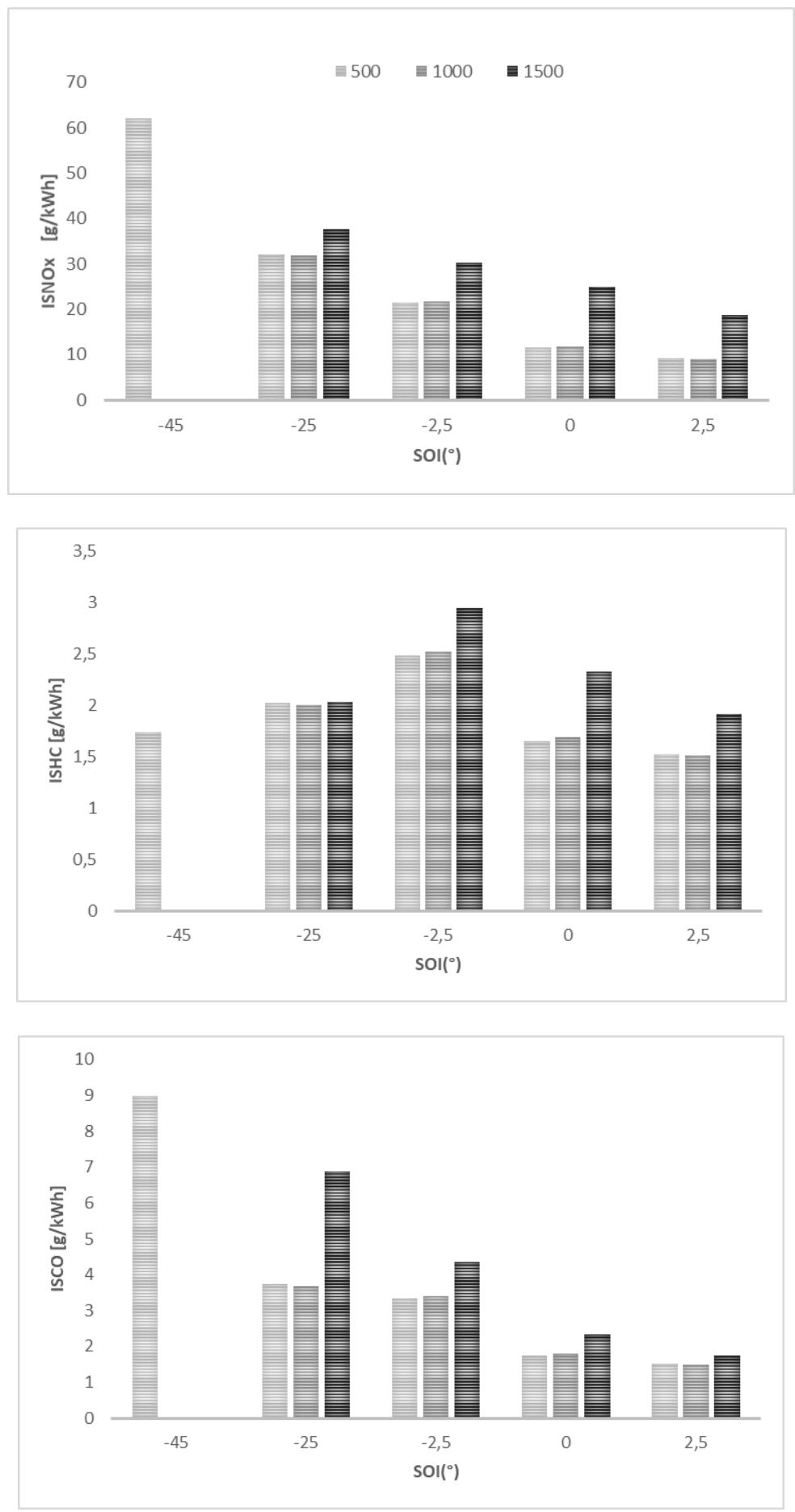


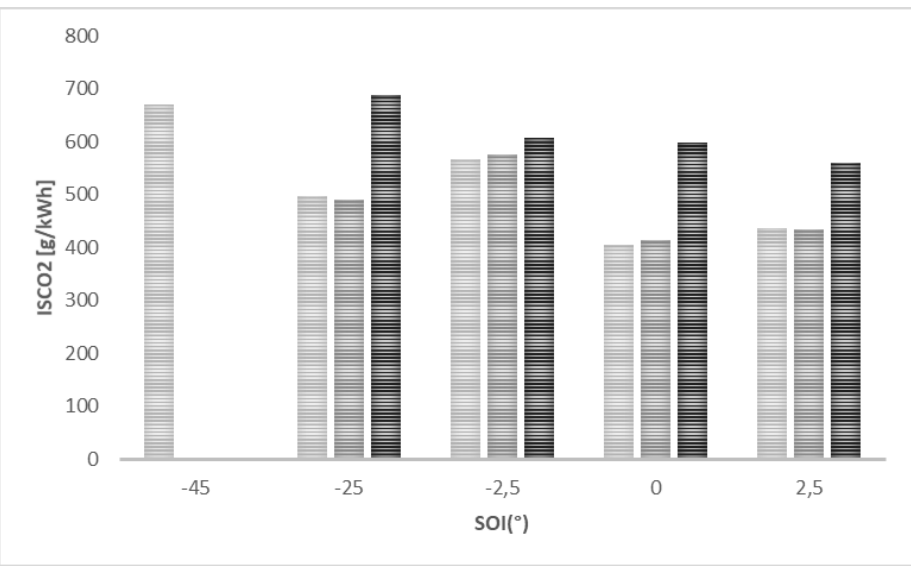

Fig. 6. Emission at 500bar and $\mathrm{C}$ condition

\subsection{Engine efficiency at different SOI}

As previously said, the combustion process parameters were calculated starting from the incylinder pressure. The fuel conversion efficiency is illustrated in Fig.7, varying the SOI at different injection pressures for high methane amount.

The engine efficiency is constant if referred to the first SOI range (advanced SOI) while it increases around -2.5 CAD (BTDC) and $0 \mathrm{CAD}$. Pressure injection also, permit to increase the engine efficiency, if compare to the other pressure at different SOI.

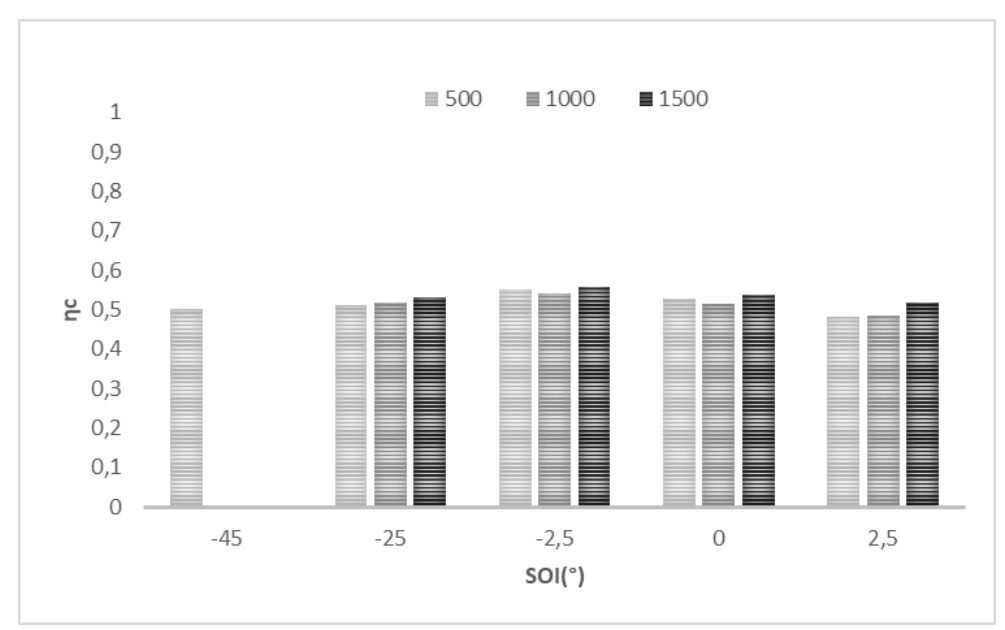

Fig. 7. Efficiency of thermodynamic cycle at c) condition.

This underlines the positive aspects of MK combustion and permits to define, in relation to the others parameters, the SOI range where quality combustion is higher (from -2.5 CAD (BTDC) to 0 CAD). 


\section{CONCLUSIONS}

In this study the late injection assessment and engine behavior for dual fuel mode have been investigated by means of experimental tests performed using a single cylinder, 4-stroke, common-rail Diesel research engine.

Some misfire points were detected during the tests, for injection pressure of 1000 and 1500 bar with very advanced SOI and late SOI, as a result of a local reduction of air-fuel ratio under the stoichiometric value, due to a faster and better vaporization of fuel. Based on the behavior of the combustion, two different SOI ranges were identified. The first (advanced SOI) characterized by a premixed combustion with a small diffusive combustion at the end of the combustion process, the second (late SOI) with a HRR shape closer to the one observed in conventional diesel combustion. A HRR peak increase has been observed as SOI was retarded from very advanced SOI, while it decreased as SOI was further retarded after TDC, for any methane rate considered. Furthermore, an increase of HRR peak has been observed by increasing the methane rate. The start of injection had an influence also on the pollutant emissions: $\mathrm{NO}_{\mathrm{x}}$ and $\mathrm{CO}_{2}$ productions were lower at late $\mathrm{SOI}$ if compared with emissions at advanced SOI, while HC production was not significantly affected by SOI. Also energy efficiency was calculated in order to define the SOI range with high efficiency. The points characterized by a higher thermal efficiency were found in the range from -2.5 CAD (BTDC) to $0 \mathrm{CAD}$. So in this range, although a reduction of $\mathrm{NO}_{\mathrm{x}}$ and $\mathrm{CO}_{2}$ was observed, the thermal efficiency improved. This confirms that MK combustion on dual fuel engine has the potential to significantly improve the behavior of dual-fuel engines in terms of pollutant emission levels and efficiency.

For this reason, in future, the late injection could be better explored with further experimental tests. The idea is to extend this MK windows by varying other engine parameters in terms of EGR rate, swirl increase and reduction of compression ratio, that were not considered in this study. All these parameters, as shown on other studies about MK combustion on diesel engine, have a significant benefit on late injection at different engine speeds.

\section{References}

1. Alonso, A. Suarez, The Expansion of Unconventional Production of Natural Gas (Tight Gas, Gas Shale and Coal Bed Methane), s.1. : Intech, Adv. in Nat. Gas Tech., pp. 123146 (2012).

2. I.K. Muhammad, Y. Tabassam, I.K. Muhammad, F. Muhammad, W. Muhammad, Research progress in the development of natural gas as fuel for road vehicles: $A$ bibliographic review (1991-2016), Renew Sust. Energ. Rev. 66, 702-741(2016).

3. R. Pali, D. Amit, K.M Saroj, Influence of gaseous fuel induction on the various engine characteristics of a dual fuel compression ignition engine: A review. Renew. Sust. Energ. Rev. 82, 3333-3349 (2018).

4. H. Song, C. Liu, Y. Li, Z. Wang, L. Chen, X. He, J. Wang, An exploration of utilizing low-pressure diesel injection for natural gas dual-fuel low-temperature combustion, Energy 153, 248-255 (2018).

5. K. Ryu, Effects of pilot injection pressure on the combustion and emissions characteristics in a diesel engine using biodiesel-CNG dual fuel, Energ. Convers. Manage 76, 506-516 (2013).

6. K. Ryu, Effects of pilot injection timing on the combustion and emissions characteristics in a diesel engine using biodiesel-CNG dual fuel, Appl. Energ. 111, 721-751 (2013). 
7. E.S. Guerry, S.M. Raihan, K.K. Srinivasan, S.R.Krishnan, A. Sohail, Injection timing effects on partially premixed diesel-methane dual fuel low temperature combustion, Appl. Energ. 162, 99-113 (2016).

8. M.M. Ibrahim, J. Varuna Narasimhan, A. Ramesh, Comparison of the predominantly premixed charge compression ignition and the dual fuel modes of operation with biogas and diesel as fuels, Energy 89, 990-1000 (2015).

9. A. P. Carlucci, A. Ficarella, D. Laforgia, L. Strafella, Design and Calibration Strategies for Improving HCCI Combustion in Dual-Fuel Diesel-Methane Engines, Natural Gas Engines, pp.267-296

10. S. Kimura, H. Ogawa, Y. Matsui, Y. Enomoto, An experimental analysis of lowtemperature and premixed combustion for simultaneous reduction of NOx and particulate emissions in direct injection diesel engines, Int. J. Engine Res 3, 4, pp. 249 259 (1997).

11. A. Carlucci, D. Laforgia, R. Saracino, G. Toto, Study of Combustion Development in Methane-Diesel Dual Fuel Engines, Based on the Analysis of In-Cylinder Luminance, SAE Tech. Paper 2010-01-1297 (2010). 